\title{
ORIGINAL ARTICLE \\ Chronic abdominal pain in long-term spinal cord injury: a follow-up study
}

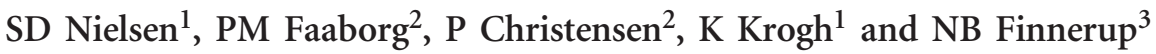

Study design: A longitudinal postal survey.

Objectives: To evaluate the prevalence and characteristics of abdominal pain in long-term spinal cord injury (SCl).

Setting: Members of the Danish SCl Association.

Methods: In 2006, a questionnaire on chronic abdominal pain and discomfort was sent to the 284 members of the Danish SCl association who had been members for at least 10 years; 203 of them responded. An almost identical questionnaire including questions on intensity and interference of pain within the past 7 days, as well as descriptors and treatment, was sent to the 178 surviving members in 2015.

Results: Of 130 (73\%) responders, 125 answered the question on chronic abdominal pain. The mean time since injury was 30.5 (9.8) years. Chronic abdominal pain or discomfort was reported by 32.8\% (41/125), and 23\% (29/125) of responders had been at least moderately bothered by this in the past week. Abdominal pain or discomfort was more common in women and in those with self-reported constipation. The median intensity (numeric rating scale) was 6.0 (range 3-10) and it was often associated with autonomic symptoms. Nine (8\%) of the 115 individuals who responded in both 2006 and 2015 had developed new abdominal pain or discomfort, $30(26 \%)$ no longer reported it, and 28 (24\%) reported it at both time points with a similar intensity.

Conclusions: Chronic abdominal pain or discomfort is common and bothersome in long-term SCl. It has a late onset, but the prevalence and severity do not seem to further increase between 20 and 30 years following SCl.

Spinal Cord (2017) 55, 290-293; doi:10.1038/sc.2016.124; published online 9 August 2016?

\section{INTRODUCTION}

Spinal cord injury (SCI) is often associated with different types of chronic pain severely affecting rehabilitation, mood, sleep, social integration and quality of life $^{1-4}$ Chronic abdominal pain is common in SCI individuals with long-term injury, is often severe and is among the most common problems that impair the quality of life in those with gastrointestinal problems. ${ }^{5-8}$ It is often characterized as a dull, diffuse, cramping pain associated with nausea and sweating. ${ }^{6,9-10}$ The characteristics of abdominal pain in SCI resemble those of chronic idiopathic constipation. ${ }^{8}$ Abdominal pain is not associated with completeness or the level of $\mathrm{SCI},{ }^{5,7}$ and the underlying mechanisms are likely to vary among SCI individuals and may be related to both visceral and neuropathic pain mechanisms. ${ }^{6-13}$

Abdominal pain has a late onset after SCI, and although it is relatively uncommon in the first 5 years, ${ }^{2,4-5}$ it has been reported in $22 \%$ at 10 years and in up to $34 \%$ of SCI individuals at 20 years after SCI. ${ }^{5-6}$ However, little is known about the time course of abdominal pain once it has developed.

The aim of the present follow-up questionnaire study was to evaluate the prevalence and time course of abdominal pain, and discomfort in long-term SCI, to characterize abdominal sensory symptoms and to examine the relationship between gastrointestinal function and abdominal pain.

\section{SUBJECTS AND METHODS}

Subjects

This study was part of a prospective questionnaire study examining bowel function in individuals with SCI. The first questionnaire was sent to all members of the Danish SCI organization 'RYK' in 1996. In a follow-up study of the same population in 2006, 284 members were sent the same questionnaire in addition to a questionnaire addressing abdominal pain or discomfort. Of them, 203 responded. ${ }^{6}$ In the current study, 178 of the original 589 members were alive and still members of the organization, and were sent two questionnaires, again addressing abdominal function and abdominal pain. The study was conducted between 24 September and 1 December 2015.

Results on abdominal function are published elsewhere (Nielsen et al., unpublished).

\begin{abstract}
Assessments
The present questionnaire on abdominal pain and discomfort included nine items and was nearly identical to the one used in 2006. Participants were first asked whether they had experienced chronic abdominal pain or discomfort, defined as pain being present constantly or at least once a week for at least 3 months. Those with chronic pain or discomfort localized in the abdomen were then asked how much this had bothered them in the past week (not at all, a little, moderately or much bothered). ${ }^{6}$ Individuals who had been bothered by pain or discomfort were asked additional questions. The average intensity was assessed on a numeric rating scale (NRS) from 0 to 10 and they filled out a list of 18 possible pain descriptors using the brief Danish Pain Questionnaire. ${ }^{14}$ Other questions addressed temporal aspects,
\end{abstract}

${ }^{1}$ Department of Hepatology and Gastroenterology, Neurogastroenterology Unit, Aarhus University Hospital, Aarhus, Denmark; ${ }^{2}$ Department of Surgery, Pelvic Floor Unit, Aarhus University Hospital, Aarhus, Denmark and ${ }^{3}$ Department of Clinical Medicine, Danish Pain Research Center, Aarhus University Hospital, Aarhus, Denmark

Correspondence: Professor NB Finnerup, Department of Clinical Medicine, Danish Pain Research Center, Aarhus University Hospital, Noerrebrogade 44, Building 1A, DK-8000 Aarhus C, Denmark.

E-mail: finnerup@clin.au.dk

Received 18 May 2016; revised 30 June 2016; accepted 1 July 2016; published online 9 August 2016 
concomitant symptoms, alleviating and aggravating factors, and treatment. ${ }^{6}$ Questions about age, gender, time since SCI and abdominal functioning were also included. Nonresponders were sent a reminder after 3 weeks.

\section{Statistical analysis}

All data were entered using EpiData EntryClient (EpiData Association, Odense, Denmark) and each entry was double-checked to reduce the risk of typing errors (IBM, Armonk, NY, USA). Statistical analyses were performed using SPSS, version 13. Study variables were described by mean and s.d., or median (range). We used Pearson $\chi^{2}$-test and the independent samples $t$-test to analyze differences between groups, and the McNemar's test and the Wilcoxon test to analyze paired data (comparison between results from 2006 and 2015). $P<0.05$ was considered statistically significant. Missing data were not replaced.

\section{RESULTS}

Subjects

Of the 178 members who were sent a questionnaire in 2015, 130 (73\%) responded. Only 125 of the 130 responders answered the question on chronic abdominal pain and were included in the analyses. Of them, 115 had also answered the questionnaire in 2006.

\section{Abdominal pain}

Chronic abdominal pain or discomfort was reported by $41 / 125$ $(32.8 \%)$ responders. Of them, 1 had not been bothered in the past week, 9 (22\%) had been bothered a little, 13 (32\%) moderately and 16 (39\%) a lot (2 did not respond). Of all 125 responders, 23\% had been at least moderately bothered by chronic abdominal pain in the past week. Abdominal pain or discomfort was more common in women $\left(P=0.038\right.$, Pearson $\chi^{2}$-test) and was related to constipation (Table 1). Among the 40 individuals who were bothered by pain or discomfort in the past week, the median intensity (NRS) was 6.0 (range 3-10). Eight (20\%) individuals reported constant abdominal pain, 13 (33\%) daily abdominal pain, $9(22 \%)$ at least 3 times per week and $8(20 \%) 1-2$ times per week. Fourteen individuals (35\%) reported receiving treatment for abdominal pain, 4 reported taking drugs for constipation and 1 for bladder problems, and 9 reported taking tramadol or opioids, of whom 6 also took paracetamol, 1 also took nortriptyline and 2 pregabalin.

Abdominal pain or discomfort was increased by constipation in $73 \%$ of responders, by full bladder in $68 \%$, by bladder infection in $67 \%$, by stress in $55 \%$, by food intake in $52 \%$ and by defecation in $29 \%$. Defecation relieved pain in $51 \%$ and bladder emptying in $39 \%$.

Table 1 Demographics of the subjects responding in 2015

\begin{tabular}{|c|c|c|c|c|}
\hline Item & All subjects & With abdominal pain or discomfort & Without abdominal pain or discomfort & $\mathrm{P}$-value $\mathrm{a}^{\mathrm{a}}$ \\
\hline Number & 130 & $41 / 125(33 \%)$ & $84 / 125(67 \%)$ & \\
\hline \multicolumn{5}{|l|}{ Gender, n (\%) } \\
\hline Female & $36(28)$ & $16(39)$ & $18(21)$ & \multirow[t]{2}{*}{$0.038^{b}$} \\
\hline Male & $94(72)$ & $25(61)$ & $66(79)$ & \\
\hline Age, years, mean (s.d.) & $56.0(9.8)$ & $53.6(8.1)$ & $56.5(10.0)$ & $0.12^{\mathrm{c}}$ \\
\hline Range & 35-82 & & & \\
\hline Time since injury, years, mean (s.d.) & $30.5(9.8)$ & $29.5(9.8)$ & $30.9(8.6)$ & $0.43^{c}$ \\
\hline Range & 20-69 & & & \\
\hline \multicolumn{5}{|l|}{ Self-reported constipation, $n(\%)$} \\
\hline Not at all & $33(25)$ & $4(10)$ & $27(32)$ & \multirow[t]{5}{*}{$0.001^{\mathrm{b}}$} \\
\hline A little & $55(42)$ & $18(44)$ & $36(43)$ & \\
\hline Some & $30(23)$ & $10(24)$ & $19(23)$ & \\
\hline A lot & $11(9)$ & $9(22)$ & $2(2)$ & \\
\hline Unknown & $1(8)$ & - & - & \\
\hline \multicolumn{5}{|l|}{ Defecation frequency, $n(\%)$} \\
\hline Every or every other day & $111(85)$ & $36(88)$ & $71(85)$ & \multirow[t]{3}{*}{$0.62^{b}$} \\
\hline Less than every other day & $18(14)$ & $5(12)$ & $13(16)$ & \\
\hline Unknown & $1(1)$ & - & - & \\
\hline \multicolumn{5}{|l|}{ Time needed for defecation, $n(\%)$} \\
\hline Up to $15 \mathrm{~min}$ & $46(35)$ & $8(20)$ & $36(43)$ & \\
\hline$>15 \min$ & $77(59)$ & $32(78)$ & $45(54)$ & \\
\hline Unknown & $7(5)$ & $1(2)$ & $3(4)$ & $0.029^{b}$ \\
\hline \multicolumn{5}{|l|}{ Constipation affects QoL, $n(\%)$} \\
\hline Not at all & 64 (49) & $10(24)$ & $53(63)$ & \multirow[t]{5}{*}{$<0.001^{\mathrm{b}}$} \\
\hline A little & $32(25)$ & $12(29)$ & $19(23)$ & \\
\hline Some & $20(15)$ & $12(29)$ & $8(10)$ & \\
\hline A lot & $9(7)$ & 7 (17) & $2(2)$ & \\
\hline Unknown & $5(4)$ & - & $2(2)$ & \\
\hline
\end{tabular}

Abbreviation: QoL, quality of life.

Only 125 individuals responded to the question on chronic abdominal pain.

${ }^{a}$ Comparison between individuals with and those without pain.

bPearson $\chi^{2}$.

Independent-sample $t$-test. 


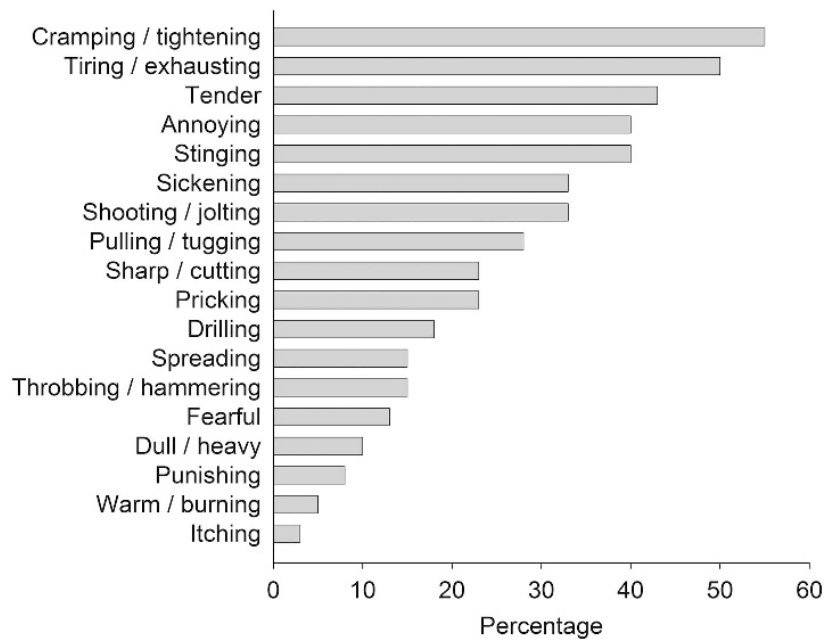

Figure 1 Descriptors of abdominal pain or discomfort on the Brief Danish Pain Questionnaire ${ }^{14}$ reported by 41 patients.

Twenty-three individuals (60\%) reported concomitant symptoms, which were sweating in 11 , nausea in 9 , headache in 2 , hunger in 2 , and spasms, high pulse, dizziness and tiredness in 1 each. The sensory descriptors were similar to those reported in 2006, with cramping, tiring, tender, and annoying being the most common (Figure 1).

\section{Abdominal pain over time \\ The percentage of responders with abdominal pain in $2015(32.8 \%)$ was almost identical to the percentage in 2006 (34.2\%), and the median pain intensity was the same (6.0). ${ }^{6}$ One hundred and fifteen individuals responded to the question on pain in both 2006 and 2015, and they were further compared. Twenty-eight individuals $(24 \%)$ had abdominal pain or discomfort at both time points, and $48(42 \%)$ did not have abdominal pain or discomfort at either time point. Nine (8\%) individuals had developed pain in 2015 that was not present in 2006, and 30 (26\%) individuals with abdominal pain in 2006 no longer reported abdominal pain in 2015 (Table 2). Therefore, although the overall prevalence was the same, the proportion who reported abdominal pain or discomfort among those responding to both questionnaires decreased from $50 \%$ in 2006 to $32 \%$ in 2015 ( $P=0.001$, McNemar's test), because more individuals reporting abdominal pain or discomfort in 2006 responded in 2015. There was no difference in median intensity of pain and discomfort among those presenting with this at both time periods (19/28 answers, $P=0.24$, Wilcoxon test). Among those responding to both the questionnaires, the proportion who used more than $15 \mathrm{~min}$ to defecate and the proportion who reported that constipation had an effect on their quality of life also had not changed from 2006 to 2015 ( $P>0.79$, McNemar's test), but the use of laxatives increased from $32 \%$ in 2006 to $52 \%$ in $2015(P<0.001$, McNemar's test).}

\section{DISCUSSION}

This follow-up questionnaire study showed that chronic abdominal pain or discomfort was present in $32.8 \%$ of responders, and thus continued to be prevalent and bothersome on average 30 years after SCI. In many patients, abdominal pain or discomfort was still present with the same intensity as in 2006, but in half of the individuals with pain or discomfort in 2006, this was no longer present in 2015. In
Table $2 \mathrm{SCl}$ individuals who reported abdominal pain or discomfort in 2006 and 2015

\begin{tabular}{lccc}
\hline & Pain 2015 & No pain 2015 & \\
\hline Pain 2006 & $28(24 \%)$ & $30(36 \%)$ & $58(50 \%)$ \\
No pain 2006 & $9(8 \%)$ & $48(42 \%)$ & $57(50 \%)$ \\
& $37(32 \%)$ & $78(68 \%)$ & 115 \\
\hline
\end{tabular}

Abbreviation: $\mathrm{SCl}$, spinal cord injury.

comparison, there was no significant change in gastrointestinal symptoms related to constipation, although the consumption of laxatives had increased (Nielsen et al., unpublished). The intensity of abdominal pain or discomfort was high with a median intensity of 6.0 on an NRS from 0 to 10 , and of all responders, $23 \%$ had been at least moderately bothered by chronic abdominal pain in the past week.

The reported sensory descriptors for abdominal pain or discomfort were similar to those used in 2006, with cramping, tiring, tender and annoying being the most common. More than half of the individuals reported concomitant symptoms of mainly autonomic character during periods with abdominal pain or discomfort. Similar to the findings in the 2006 questionnaire, abdominal pain or discomfort was related to constipation with a higher frequency of self-reported constipation and a higher impact of constipation on the quality of life. In addition, constipation was reported to increase abdominal pain or discomfort in $73 \%$ and food intake in $55 \%$ of responders. Also, having a full bladder and bladder infection often increased abdominal pain or discomfort. In contrast to the findings in 2006, ${ }^{6}$ more individuals with abdominal pain or discomfort reported that they needed $>15 \mathrm{~min}$ for defecation, whereas there was no difference in defecation frequency. Also, we found infrequent use of typical neuropathic pain characteristics such as pricking and burning pain. This supports previous suggestions that abdominal pain or discomfort is related to constipation and abnormal gastrointestinal function in the majority of SCI individuals. ${ }^{6,8}$ The effect of a bowel program on different neurogenic bowel symptoms including abdominal pain supports the relation between abdominal pain and constipation. ${ }^{13}$ However, it is possible that neuropathic pain contributes to abdominal pain in some of the patients.

Opioids were the most common treatment reported for abdominal pain. If constipation is the main underlying cause, the use of opioids may be a disadvantage for SCI individuals and may even worsen their pain. A careful evaluation of the pain, gastrointestinal function and intestinal dysmotility as well as sensory function is important, and treatment aimed at improving gastrointestinal function and neurogenic bowel disorders may be the most appropriate treatment for abdominal pain. Such treatment could include laxatives, prokinetics, ${ }^{15}$ transanal irrigation, ${ }^{16}$ appendicostomy ${ }^{17}$ or colostomy/ ileostomy. ${ }^{18}$

We had a response rate of $73 \%$, and a comparison with the results from 2006 suggested that those with abdominal pain or discomfort in 2006 were more likely to respond again in 2015, suggesting an overestimation of the estimated prevalence. However, a comparison of the results at the two time points showed that, despite the similar overall percentages, there were more SCI individuals who had discontinued than those who had developed chronic abdominal pain or discomfort over the 10-year period. The lack of a detailed pain history and clinical examination is another limitation of the study. 


\section{CONCLUSION}

Chronic pain or discomfort located in the abdomen is common in chronic SCI and persists in a large proportion of individuals. It seems to be mainly related to constipation.

\section{DATA ARCHIVING}

There were no data to deposit.

\section{CONFLICT OF INTEREST}

The authors declare no conflict of interest.

1 Sezer N, Akkus S, Ugurlu FG. Chronic complications of spinal cord injury. World J Orthop 2015; 6: 24-33.

2 Finnerup NB, Jensen MP, Norrbrink C, Trok K, Johannesen IL, Jensen TS et al. A prospective study of pain and psychological functioning following traumatic spinal cord injury. Spinal Cord 2016; 54: 816-821.

3 Andresen SR, Biering-Sørensen F, Hagen EM, Nielsen JF, Bach FW, Finnerup NB. Pain, spasticity and quality of life in individuals with traumatic spinal cord injury in Denmark. Spinal Cord 2016; 54: 973-979.

4 Siddall PJ, McClelland JM, Rutkowski SB, Cousins MJ. A longitudinal study of the prevalence and characteristics of pain in the first 5 years following spinal cord injury. Pain 2003; 103: 249-257.

5 Kogos SC Jr, Richards JS, Baños JH, Ness TJ, Charlifue SW, Whiteneck GG et al. Visceral pain and life quality in persons with spinal cord injury: a brief report. J Spinal Cord Med 2005; 28: 333-337.

6 Finnerup NB, Faaborg P, Krogh K, Jensen TS. Abdominal pain in long-term spinal cord injury. Spinal Cord 2008; 46: 198-203.
7 Stone JM, Nino-Murcia M, Wolfe VA, Perkash I. Chronic gastrointestinal problems in spinal cord injury patients: a prospective analysis. Am J Gastroenterol 1990; 85 1114-1119.

8 Faaborg PM, Finnerup NB, Christensen P, Krogh K. Abdominal pain: a comparison between neurogenic bowel dysfunction and chronic idiopathic constipation. Gastroenterol Res Pract 2013; 2013: 365037.

9 Ebert E. Gastrointestinal involvement in spinal cord injury: a clinical perspective. J Gastrointestin Liver Dis 2012; 21: 75-82.

10 Bryce TN, Biering-Sørensen F, Finnerup NB, Cardenas DD, Defrin R, Lundeberg T et al. International spinal cord injury pain classification: part I. Background and description. Spinal Cord 2012; 50: 413-417.

11 McKibben MJ, Seed P, Ross SS, Borawski KM. Urinary tract infection and neurogenic bladder. Urol Clin North Am 2015; 42: 527-536.

12 Park HK, Song DH, Kim YM, Kim KG, Kim SY, Chung ME. Intractable abdominal pain in a patient with spinal cord injury: a case report. Ann Rehabil Med 2013; 37: 721-724.

13 Ozisler Z, Koklu K, Ozel S, Unsal-Delialioglu S. Outcomes of bowel program in spinal cord injury patients with neurogenic bowel dysfunction. Neural Regen Res 2015; 10: 1153-1158.

14 Perkins FM, Werner MU, Persson F, Holte K, Jensen TS, Kehlet H. Development and validation of a brief, descriptive Danish pain questionnaire (BDDPQ). Acta Anaesthesiol Scand 2004; 48: 486-490.

15 Krogh K, Jensen MB, Gandrup P, Laurberg S, Nilsson J, Kerstens R et al. Efficacy and tolerability of prucalopride in patients with constipation due to spinal cord injury. Scand J Gastroenterol 2002; 37: 431-436.

16 Christensen P, Bazzocchi G, Coggrave M, Abel R, Hultling C, Krogh K et al. A randomized, controlled trial of transanal irrigation versus conservative bowel management in spinal cord-injured patients. Spinal Cord 2006; 131. 738-747.

17 Worsøe J, Christensen P, Krogh K, Buntzen S, Laurberg S. Long-term results of antegrade colonic enema in adult patients: assessment of functional results. Dis Colon Rectum 2008; 51: 1523-1528.

18 Coggrave MJ, Ingram RM, Gardner BP, Norton CS. The impact of stoma for bowel management after spinal cord injury. Spinal Cord 2012; 50: $848-852$. 